\title{
BURNOUT Y SU RELACIÓN CON RASGOS DE PERSONALIDAD EN UNA MUESTRA DE MÉDICOS DEL SUR DEL ECUADOR
}

\author{
Marina R. Ramírez \\ Universidad Técnica de Particular -Ecuador \\ mrramirez@utpl.edu.ec \\ Mercy P. Ontaneda \\ Universidad Técnica de Particular -Ecuador \\ mpontaneda@utpl.edu.ec \\ David Ortega Jímenez \\ Universidad Técnica de Particular -Ecuador \\ dmortega1@utpl.edu.ec
}

Fecha de Recepción: 17 Junio 2019

Fecha de Admisión: 25 Septiembre 2019

\section{RESUMEN}

El burnout constituye un fenómeno global, especialmente en el ámbito de profesionales de la salud por las características propias de estos profesionales supondría una mayor vulnerabilidad (Gluschkoff, Elovainio, Kinnunen, Mullola, Hintsanen, Keltikangas-Järvinen y Hintsa, 2016;). La presente investigación tuvo como objetivo identificar el burnout y la relación con los rasgos de personalidad (extroversión, psicoticismo, neuroticismo). Se utilizó una metodología descriptiva y correlacional, con una muestra de accesibilidad no probalística de 149 médicos de la zona sur del Ecuador. Los instrumentos utilizados fueron Inventario de Maslach [MBI-HSS] y el cuestionario de personalidad de Eysenck Revisado- [EPQR-A]. Entre los resultados relevantes se obtuvo una prevalencia de $2.7 \%$ del síndrome de burnout, en cuanto al agotamiento emocional $25.5 \%$ un nivel alto, en despersonalización el $10.7 \%$ un nivel alto y el 59.1\% tiene baja realización personal. Como conclusiones generales se pudo identificar una correlación significativa entre neuroticismo y el burnout y con las dimensiones agotamiento emocional; despersonalización, así como también entre extroversión y despersonalización.

Palabras claves: burnout; agotamiento emocional; despersonalización; baja realización personalidad; neuroticismo; extraversión; psicoticismo.

\section{ABSTRACT \\ Burnout and its relationship with personality risks in a sample of doctors from south Ecuador. Burnout is a global phenomenon, especially in the field of health professionals because of the characteristics of these professionals would be more vulnerable (Gluschkoff, Elovainio, Kinnunen,}




\section{BURNOUT Y SU RELACIÓN CON RASGOS DE PERSONALIDAD EN UNA MUESTRA DE MÉDICOS DEL SUR DEL ECUADOR}

Mullola, Hintsanen, Keltikangas-Järvinen and Hintsa, 2016). The objective of this research was to identify burnout and the relationship with personality traits (extroversion, psychoticism, neuroticism). A descriptive and correlational methodology was used, with a non-probalistic accessibility sample of 149 physicians from the southern area of Ecuador. The instruments used were the Maslach Inventory [MBI-HSS] and the Eysenck Revised Personality Questionnaire- [EPQRA]. Among the relevant results, a $2.7 \%$ prevalence of burnout syndrome was obtained, in terms of emotional exhaustion $25.5 \%$ a high level, in depersonalization $10.7 \%$ a high level and $59.1 \%$ have low personal achievement. As general conclusions we could identify a significant correlation between neuroticism and burnout and emotional exhaustion dimensions; depersonalization, as well as between extroversion and depersonalization.

Keywords: burnout; emotional exhaustion; depersonalization; low personality realization; neuroticism; extraversion; psychoticism.

\section{ANTECEDENTES DE LA TEMÁTICA A TRATAR}

El término burnout se empezó a utilizar hace más de cinco décadas en relación al desgaste profesional que sufrían las personas que su ambiente laboral. Al respecto, Maslach y Jackson (1986) explican que es una condición psicológica que implica una respuesta prolongada a estresores interpersonales en el trabajo. Además, establecen como un síndrome tridimensional caracterizado por:

Agotamiento emocional sentimientos de estar agotado, sobre exigido y con falta de recursos emocionales y físicos para afrontar esta situación llegando a carecer de energía, a este acontecimiento principalmente se atribuye la sobrecarga laboral y el conflicto en el trabajo.

Despersonalización: actuación de las personas de manera negativa en el trabajo, lo que hará que no cumpla sus actividades normales disminuyendo su desempeño laboral, también lo reconocen como cinismo que refiere a una respuesta insensible o excesivamente apática a diversos aspectos del trabajo y se desarrolla frente al fracaso para enfrentar de manera positiva el agotamiento emocional.

Baja realización personal: sugiere que en base a las propias auto valoraciones las personas piensan que no están cumpliendo con sus expectativas sobre el desempeño en su trabajo.

Hasta el momento, aún no se ha llegado a un consenso en la comunidad científica para la traducción al español del constructo del burnout ya que es un término anglosajón, en este sentido GilMonte y Moreno-Jiménez (2007) indican lo siguiente:

La revisión de la literatura nos ofrece al menos diecisiete denominaciones diferentes en español para el fenómeno, si bien algunas de ellas presentan una gran similitud. Considerando ese grupo de similitud, las denominaciones pueden ser clasificadas en tres grupos: a) denominaciones que toman como referencia el término original anglosajón "Burnout" (síndrome de quemarse por el trabajo, síndrome de estar quemado en el trabajo, síndrome de quemarse en el trabajo, síndrome de estar quemado, síndrome del quemado, estar quemado y quemazón profesional); b) denominaciones que toman como referencia el contenido semántico de la palabra, o el contenido de la patología (desgaste psicológico por el trabajo, desgaste ocupacional, desgaste profesional, agotamiento profesional, agotamiento laboral y síndrome de cansancio emocional), y c) estudios en los que se considera que el síndrome de quemarse por el trabajo es sinónimo de estrés laboral (estrés crónico laboral asistencial, estrés laboral asistencial, estrés profesional y estrés laboral) (p. 21).

Consideremos importante desarrollar la propuesta en los profesionales de la salud en función de ser considerados como una población vulnerable propensos a estresarse, como el ambiente laboral, en el que deben desempeñarse con gran responsabilidad, además, en muchos casos los profesionales se plantean metas que no pueden alcanzar, consecuentemente se presenta la frustración por el rol que desempeña (Organización Mundial de Salud [OMS] 2000). 
En los últimos años los casos del burnout en los profesionales de la salud han incrementado en un número considerable, lo que se determina que es urgente actuar para frenar estas cifras, de manera especial sugieren prestar atención a las políticas de las instituciones y la preparación previa de los trabajadores de la salud (Regal, 2016). En cuanto al tema Herrera et al. (2016) menciona que en los últimos años ha investigado la prevalencia del Síndrome de Burnout, conocido como Síndrome del Trabajador Quemado, y el Engagement o Compromiso, en diferentes profesiones, destacando las sanitarias.

Por su parte, Pérez (2013) indica la importancia de enfocar los estudios del burnout en los medios hospitalarios, aquello debido a la mejora de las situaciones adversas para propiciar ambientes que favorezcan el desenvolvimiento de los profesionales, recalca la propia naturaleza del trabajo que trae consigo sentimientos de incertidumbre, los mismos deben ser apoyados, para modificarlos. Al respecto Cruz, Nelas, Coutinho, Chaves, \& Amaral (2018) mencionan que los cambios en la carrera profesional y las condiciones de trabajo implican un compromiso de la salud física y mental, con perjuicio en la satisfacción, realización y burnout en los profesionales de la salud específicamente en los enfermeros.

Diversos autores han investigado las características de personalidad y el burnout tratando de identificar que rasgos de personalidad hacen a los sujetos más vulnerables a desarrollar el burnout. Es así que McManus, Jonvik, Richards, y Paice (2011) mencionan que el agotamiento emocional, aumenta el grado de neuroticismo y la extroversión. Al respecto Cebriá y et al. (2001); en una investigación en médicos en el que se analizó los rasgos de personalidad y el síndrome de burnout, encontró asociaciones significativas con la baja estabilidad emocional, tensión y ansiedad con las altas puntuaciones del burnout. Las personas con un alto neuroticismo presentan mayor cansancio emocional y despersonalización (Olmedo Montes, Germán, Jiménez Tornero y Castillo, 2001). Otros autores han encontrado un mayor riesgo de burnout en trabajadores con personalidad ansiosa (Jaracz, Rosiak, Bertrand-Buci ska, Jaskulski, Nie urawska y Borkowska, 2017), o con un estilo personal dominante en sus relaciones interpersonales (Martínez-Zaragoza, 2018).

\section{OBJETIVOS DE LA INVESTIGACIÓN}

\section{Objetivo general}

Identificar la relación entre el síndrome de burnout y los rasgos de personalidad en una muestra de médicos del sur del Ecuador

\section{Objetivos Específicos}

Determinar la prevalencia del síndrome de burnout y de las tres dimensiones: agotamiento emocional, despersonalización y baja realización personal.

Identificar los rasgos de personalidad de la muestra de investigación

Relacionar el síndrome de burnout con los rasgos de personalidad de los médicos.

\section{MUESTRA Y/O PARTICIPANTES}

La muestra fue de accesibilidad-accidental y no probabilística ya que se seleccionan de manera casual, es decir de acuerdo al medio idóneo para ejecutar el estudio y busca los datos en función de los objetivos de la investigación (Hernández Sampieri, Fernández Collado y Baptista Lucio, 2010), finalmente la muestra recolectora de datos fue 149 médicos/as.

El $51 \%$ hombres y el $49 \%$ mujeres, la edad promedio de 40.64 comprendido entre 27 y 64 años, además se evidencia una media de 1.7 hijos, por otro lado, el 96\% pertenecía a la etnia mestiza, finalmente, el $63.1 \%$ estaban casados, seguido por el $20.8 \%$ que eran solteros. En lo referente a las 


\section{BURNOUT Y SU RELACIÓN CON RASGOS DE PERSONALIDAD EN UNA MUESTRA DE MÉDICOS DEL SUR DEL ECUADOR}

variables laborales el $47.7 \%$ percibía un sueldo entre 1001 y 2000 , en cuanto a la experiencia laboral, presentan un promedio de 13.5 años, considerando las horas de trabajo diarias se presenta una media de 10.1 horas respecto al número de pacientes por día se presentó una media aproximada de 20 pacientes al día.

Los criterios considerados para seleccionar la muestra fueron:

Criterios de inclusión: Ser profesional de medicina, situación activa, firmar el consentimiento, tener 2 años mínimo de experiencia.

Criterios de exclusión: Ser técnico o auxiliar de medicina, no firmar el consentimiento informado.

\section{METODOLOGÍA Y/O INSTRUMENTOS UTILIZADOS}

El diseño de la investigación es transversal de tipo descriptivo y correlacional con un enfoque cuantitativo. Al respecto Hernández Sampieri, Fernández Collado y Baptista Lucio (2010) señalan que el estudio transversal permite que los datos se recolecten en un solo momento y tiempo único, refiriendo al estudio descriptivo es aquel que busca especificar propiedades, características y rasgos importantes del fenómeno a estudiar, en cuanto al estudio correlacional, evalúa la relación entre variables y en lo que refiere al tipo cuantitativo, se enfoca en analizar datos cuantitativos sobre las variables, mediante herramientas estadísticas.

Inventario de burnout de Maslach (Maslach burnout Inventory [MBI]; Maslach y Jackson, 1986; versión española de Seisdedos, 1997)

Este instrumento ha sido diseñado para valorar el síndrome de burnout en sus tres aspectos fundamentales: cansancio emocional, despersonalización y baja realización personal. Está compuesto por 22 ítems. Para las respuestas se utiliza una escala de Likert con puntuaciones de 0 a 6 , de forma que 0 supone que lo expuesto no sucede nunca y 6 que sucede todos los días, dejando el resto de puntuaciones intermedias para la frecuencia de aparición situadas entre estos dos extremos.

La subescala de cansancio emocional está compuesta por 9 ítems y mide los sentimientos de una persona emocionalmente exhausta por el propio trabajo.. La subescala de despersonalización está integrada por 5 ítems y valora los sentimientos y actitudes negativas hacia el paciente. La subescala de realización personal consta de 8 ítems y mide sentimientos de competencia y de éxito en el trabajo. En cuanto a las propiedades psicométricas, la consistencia interna (alfa de Cronbach) del MBI oscila en el rango de .70 a .90 (Maslach y Jackson, 1986).

Cuestionario de Personalidad de Eysenck Revisado-Abreviado (Eysenck Personality Questionnaire Revised-Abbreviated [EPQR-A]); Francis, Brown y Philipchalk, 1992; versión española de Sandín, Valiente y Chorot, 1999)

El EPQR-A consta de 24 ítems y cuatro subescalas (neuroticismo, extraversión, psicoticismo y sinceridad), con 6 ítems por cada una de ellas (ver Anexo 6). Las tres primeras subescalas miden rasgos de personalidad, mientras que la última evalúa la tendencia a mentir ( 0 a emitir respuestas de deseabilidad social; en la versión española, al igual que ocurre con el cuestionario EPQ, se evalúa inversamente, esto es, se calcula el nivel de sinceridad). El formato de respuesta es de Sí (1) vs. No (0), con un rango de puntuaciones para cada subescala entre 0 y 6 , interpretándose que a mayor puntuación en cada rasgo mayor presencia del mismo.

Sandín, Valiente, Montes, Chorot y Germán (2002) hallaron una consistencia interna (alfa de Cronbach) para la extraversión de 0.74 , para el neuroticismo de 0.78 , para el psicoticismo de 0.63 y para la sinceridad de 0.54 . 


\section{RESULTADOS}

Tabla 1 Dimensiones del Burnout en los médicos

\begin{tabular}{llrl}
\hline \hline & & $n$ & $\%$ \\
\hline Agotamiento emocional & Bajo & 83 & 55.7 \\
& Medio & 28 & 18.8 \\
& Alto & 38 & 25.5 \\
Despersonalización & Bajo & 100 & 67.1 \\
& Medio & 36 & 22.1 \\
& Alto & 16 & 10.7 \\
Realización personal & Alto & 27 & 18.1 \\
& Medio & 34 & 22.8 \\
& Bajo & 88 & 59.1 \\
\hline \hline
\end{tabular}

Fuente: Inventario de Burnout de Maslach (Maslach Burnout Inventory [MBI];

Maslach y Jackson, 1986)

Refiriendo a la prevalencia de la dimensión agotamiento emocional el $25.5 \%$ pertenecen a un nivel alto, en despersonalización el $10.7 \%$ corresponde al nivel alto y el $59.1 \%$ presenta baja realización personal.

Tabla 2 Casos de Burnout

\begin{tabular}{llrr}
\hline \hline & & $n$ & $\%$ \\
\hline Burnout & No presencia & 145 & 97.3 \\
& Presencia & 4 & 2.7 \\
\hline \hline
\end{tabular}

En cuanto a la prevalencia se determina que existen 4 casos del síndrome de Burnout, que corresponden al $2.7 \%$.

Tabla 3 Rasgos de Personalidad

\begin{tabular}{lccrc}
\hline & \multicolumn{4}{c}{ Médico } \\
\cline { 2 - 5 } & M & DT. & Máx. & \multicolumn{1}{c}{ Mín. } \\
\hline Neuroticismo & 2.20 & 1.79 & 6.00 & .00 \\
Extroversión & 3.92 & 1.80 & 6.00 & .00 \\
Psicoticismo & 1.81 & 1.53 & 4.00 & .00 \\
\hline
\end{tabular}

El rasgo de personalidad más alto con una media mayor fue la extroversión con 3.9, seguido de neuroticismo 2.2 y finalmente el rasgo de psicoticismo con 1.8. 


\section{BURNOUT Y SU RELACIÓN CON RASGOS DE PERSONALIDAD EN UNA MUESTRA DE MÉDICOS DEL SUR DEL ECUADOR}

Tabla 4 Correlaciones entre dimensiones de burnout y el rasgo de personalidad

\begin{tabular}{ccccccccccc}
\hline & \multicolumn{3}{c}{ Neuroticismo } & \multicolumn{3}{c}{ Extroversión } & \multicolumn{3}{c}{ Psicoticismo } \\
\cline { 2 - 10 } & $\boldsymbol{r}$ & $\boldsymbol{p}$ & $\boldsymbol{n}$ & $\boldsymbol{r}$ & $\boldsymbol{p}$ & $\boldsymbol{N}$ & $\boldsymbol{r}$ & $\boldsymbol{p}$ & $\boldsymbol{n}$ \\
\hline $\begin{array}{c}\text { Agotamiento } \\
\text { emocional }\end{array}$ & $.347^{* *}$ & .000 & 149 & .259 &. .095 & 149 & .348 & .091 & 149 \\
$\begin{array}{c}\text { Despersonalización } \\
\text { Realización } \\
\text { personal }\end{array}$ & $.313^{* *}$ & .000 & 149 & - & $.191^{*}$ & .000 & 149 & .338 & .072 & 149 \\
Burnout & -.280 & .091 & 149 & .185 & .059 & 149 & -.269 & .038 & 149 \\
& $.581^{* *}$ & .000 & 149 & .195 & .153 & 149 & .277 & .074 & 149
\end{tabular}

**. La correlación es significativa en el nivel 0,01 (bilateral).

*. La correlación es significativa en el nivel 0,05 (bilateral).

Se observa que existe una correlación muy significativa entre neuroticismo y agotamiento emocional, $r(149)=.347 p<.0001$. Existe una correlación muy significativa entre neuroticismo y despersonalización, $r(149)=.313, p<.0001$. Se evidencia una correlación muy significativa entre neuroticismo y burnout, $r(149)=.581, p<.0001$. Existe una correlación negativa entre extroversión y despersonalización, $r(149)=-.191, p=<.0001$.

\section{DISCUSIÓN}

En lo referente a las dimensiones del burnout se obtuvo en agotamiento emocional el $25.5 \%$ pertenecen a un nivel alto, al igual que en los siguientes estudios en donde los valores oscilan entre 25\% y 35\% (Apela y Bueno, 2014; Athié Gutiérrez et al., 2016; Campos, Esperanza y Machuca Mariñas, 2017; Ramírez, 2017), despersonalización el 10.7\%, otros investigadores han obtenido en sus trabajos porcentajes entre 10\% y 20\% (Adži et al., 2013; Herrera, Hernández, Fernández y Fernández, 2014; Ramírez, 2017) y el 59.1\% con baja realización personal, en otros estudios que se han referenciado están valores entre 59\% y 69\% (Silva Henao, Gutiérrez Strauss, Pando Moreno y Tuesca Molina, 2014; Piñeiro, González y Daza, 2017).

Respecto a la prevalencia del burnout fue de $2.7 \%$, al respecto existen otros estudios en los que la prevalencia oscila entre 2\% a 5\% (Arteaga-Romani, Junes-Gonzales y Navarrete-Saravia, 2018; Ramírez, 2017; Ramírez et al., 2018).

En lo referente a las correlaciones, se observa que existe una correlación muy significativa entre neuroticismo y agotamiento emocional, neuroticismo y despersonalización, así como también una relación significativa entre extroversión y despersonalización. Es así que McManus et al. (2011) mencionan que mientras aumenta el agotamiento emocional, aumenta el grado de neuroticismo. Las personas con un alto neuroticismo presentan mayor cansancio emocional y despersonalización (Olmedo Montes, Germán, et al., 2001) por lo que respecta a los rasgos de personalidad, una investigación (Swider y Zimmerman, 2010) encontró que las personas que mostraban puntuaciones más elevadas en neuroticismo y más bajas en extraversión eran más propensas a experimentar el síndrome de burnout.

\section{CONCLUSIONES}

En nuestra investigación tuvimos la participación similar en cuanto al sexo tanto hombres como mujeres profesionales en el ámbito de medicina con un promedio de edad de 40 años al menos dos hijos y mestizos y casados 
En lo referente a las caracterizaciones laborales en su mayoría percibe un sueldo en relación con la canasta básica familiar establecida para esta zona, una experiencia promedio de aproximadamente 13 años, trabaja al menos 10 horas al día y con atención de una media de 20 pacientes al día.

En relación a las dimensiones de burnout, la puntuación alta fue agotamiento emocional $25.5 \%$ pertenecen a un nivel alto, en despersonalización el $10.7 \%$ alto y el $59.1 \%$ presenta baja realización personal. La prevalencia del burnout fue de $2.7 \%$, identificándose cuatro casos de la muestra seleccionada.

En relación a los rasgos de personalidad el que obtuvo una media superior fue extroversión, seguido por neuroticismo y psicocoticismo.

En lo referente a la relación existente entre el burnout (dimensiones) y los rasgos de personalidad se identificó una relación significativa entre neuroticismo con agotamiento emocional, despersonalización y el burnout y en lo referente a la extroversión una relación negativa con despersonalización.

\section{AGRADECIMIENTO}

Realizamos un agradecimiento y mención expresa a la Asociación Universitaria Iberoamericana de Posgrado [AUIP] y a la Consejería de Economía y Conocimiento de la Junta de Andalucía, como patrocinadores del Programa iberoamericano de movilidad postdoctoral de la AUIP, ya que han sido un apoyo importante para la realización de esta investigación.

\section{REFERENCIAS BIBLIOGRÁFICAS}

Adži , Z.0. Katic, M. Kern, J. Soler, J.K. Cerove ki, V. y Polašek, 0. (2013). Is burnout in family physicians in Croatia related to interpersonal quality of care? Arhiv za higijenu rada i toksikologiju, 64 2, 69-78.

Apela, V. M. y Bueno, M. J. A. (2014). Desgaste profesional en médicos pediatras. Biomedicina, 9(1), 6-15.

Arteaga-Romani, A., Junes-Gonzales, W., \& Navarrete-Saravia, A. (2018). La prevalencia del síndrome de burnout en personal de salud. Revista Médica Panacea, 4(2), 40-44.

Athié Gutiérrez, C. Cardiel Marmolejo, L. E. Camacho Aguilera, J. Mucientes Avellaneda, V. M. Terronez Girón, A. D. M. Cabrera Mora, N. A. y Valdés Peñaloza, A. L. (2016). Burnout en médicos internos de pregrado del Hospital General de México Dr. Eduardo Liceaga. Investigación en educación médica, 5(18), 102-107.

Campos, D., Esperanza, G. y Machuca Mariñas, S. M. (2017). Dimensiones del síndrome de burnout y su presencia en el desempeño laboral del personal médico de la red de salud Cajamarca, 2017. Recuperada de http://repositorio.upagu.edu.pe/handle/UPAGU/644

Cebriá, J., Segura, J., Corbella, S., Sos, P., Comas, 0., García, M., ... \& Pérez, J. (2001). Rasgos de personalidad y burnout en médicos de familia. Atención primaria, 27(7), 459-468.

Cruz, C., Nelas, P., Coutinho, E., Chaves, C., \& Amaral, O. (2018). A SATISFAÇÃO, REALIZAÇÃO E EXAUSTÃO DOS ENFERMEIROS EM PORTUGAL. International Journal of Developmental and Educational Psychology, 3(1), 361-369.

Francis, L. J., Brown, L. B. y Philipchalk, R. (1992). The development of an abbreviated form of the Revised Eysenck Personality Questionnaire (EPQR-A): Its use among students in England, Canada, the USA and Australia. Personality and Individual Differences, 13, 443-449.

Herrera, R. C. Hernández, J. J. R. Fernández, Y. R. R. y Fernández, Y. I. R. (2014). Estrés y síndrome de Burnout en estomatólogos. Revista Cubana de Salud y Trabajo, 15(1), 42-49.

Herrera, S. S., Gordillo, M. G., Acuñas, M. G., Fernández, M. I. R., García, M. L. B., \& Méndez, M. J. 
R. (2016). El síndrome del burnout y el engagement en una muestra de dependientes de la población de Badajoz. International Journal of Developmental and Educational Psychology. Revista INFAD de Psicología., 2(1), 261-270.

Gil-Monte, P. R. y Moreno-Jiménez, B. (2007). El síndrome de quemarse por el trabajo (burnout): grupos profesionales de riesgo. Pirámide. Madrid.

Gluschkoff, K., Elovainio, M., Kinnunen, U., Mullola, S., Hintsanen, M., Keltikangas-Järvinen, L., \& Hintsa, T. (2016). Work stress, poor recovery and burnout in teachers. Occupational medicine, 66(7), 564-570.

Hernández Sampieri, R. Fernández Collado, C. y Baptista Lucio, P. (2010). Metodología de la investigación. México: McGraw-Hill.

Jaracz, M., Rosiak, I., Bertrand-Buci ska, A., Jaskulski, M., Nie urawska, J., \& Borkowska, A. (2017). Affective temperament, job stress and professional burnout in nurses and civil servants. PloS one, 12(6), e0176698.

Olmedo Montes, M., Germán, S., Jiménez Tornero, R., \& Castillo, G. (2001). El síndrome de Burnout: variables laborales, personales y psicopatológicas asociadas. Psiquis (Madr.), 22(3), 117-129.

Organización Mundial de la Salud (OMS). (2000). The World Health Report 2000. Health systems: Improving performance. Informe mundial de salud 2000. Sistemas de salud: mejorando el desempeño

Martínez-Zaragoza, F. (2018). Personality and interpersonal behaviour may impact on burnout in nurses. Evidence-based nursing, 21(1), 24.

Maslach, C. y Jackson, S. E. (1986). Maslach Burnout Inventory. 2ª edición. Palo Alto, CA: Consulting Psychologists Press.

McManus, I. C., Jonvik, H., Richards, P., \& Paice, E. (2011). Vocation and avocation: leisure activities correlate with professional engagement, but not burnout, in a cross-sectional survey of UK doctors. BMC medicine, 9(1), 100.

Piñeiro, K. González, M. y Daza, M. (2017). Sindrome de burnout en medicos de la emergencia pediatrica y unidad de medicina critica pediatrica. Investigación Clínica, 58(1 SI), 666-668.

Pérez, J.P. (2013). Efecto del burnout y la sobrecarga en la calidad de vida en el trabajo. Estudios gerenciales, 29(129), 445-455. doi: 10.1016/j.estger.2013.11.010

Ramírez, M. R. Otero, P. Blanco, V. Ontaneda, M. P. Díaz, O. y Vázquez, F. L. (2018). Prevalence and correlates of burnout in health professionals in Ecuador. Comprehensive psychiatry, 82, 73-83. doi: 10.1016/j.comppsych.2017.11.011

Ramírez, M. R. (2017). Prevalencia del síndrome de burnout y la asociación con variables sociodemográficas y laborales en una provincia de Ecuador. International Journal of Developmental and Educational Psychology. Revista INFAD de Psicología., 4(1), 241-252.

Regal, M. (2016). Vigilancia de la salud para la detección precoz del síndrome de desgaste profesional (burnout) en médicos de atención primaria. Atención Primaria, 48(4), 270-271. doi:10.1016/j.aprim.2015.02.015

Sandín, B., Valiente, R. M. y Chorot, R. (1999). Evaluación del estrés psicosocial. En B. Sandín (Ed.), El estrés psicosocial: Conceptos y consecuencias clínicas (pp. 245-316). Madrid: Klinik.

Sandín, B., Valiente, R. M., Montes, M. O., Chorot, P. y Germán, M. A. (2002). Versión española del cuestionario EPQR-Abreviado (EPQR-A) (II): Replicación factorial, fiabilidad y validez. Revista de Psicopatología y Psicología Clínica, 7, 195-205.

Seisdedos, N. (1997). MBI Inventario Burnout de Maslach. Madrid: Ediciones TEA.

Silva Henao, D. C. Gutiérrez Strauss, A. M. Pando Moreno, M. y Tuesca Molina, R. (2014). Relación 


\section{PSICOLOGIA, INFANCIA Y EDUCACIÓN}

entre factores psicosociales negativos y el síndrome de Burnout en el personal sanitario de Florencia (Caquetá, Colombia). Salud Uninorte, 30(1). 53-62.

Swider, B. W. y Zimmerman, R. D. (2010). Born to burnout: A meta-analytic path model of personality, job burnout, and work outcomes. Journal of Vocational Behavior, 76, 487-506. 
